\title{
Activity during prior shock determines subsequent shock-elicited fighting in the rat
}

\author{
D. CHRIS ANDERSON, CHARLES R. CROWELL, MARTIN B. WIKOFF, \\ and J. VICTOR LUPO \\ University of Notre Dame, Notre Dame, Indiana 46556
}

\begin{abstract}
Two experiments investigated the relationship between activity during shock and the magnitude of subsequent impairment of shock-elicited fighting in the rat. Different levels of intrashock activity were engendered in two ways. In Experiment 1, differing temporal forms of inescapable shock were employed to produce markedly different levels of activity. In Experiment 2, a passive-escape procedure was used to explicitly reinforce nonmovement during shock relative to a yoked, inescapable shock control. Results indicated that relative to the performance of subjects not previously shocked, fighting impairment was produced only by those prior treatments that promoted reduced intrashock activity. Since one of the prior shock treatments involved inescapable shock but the other did not, these findings may be viewed as strong support for the notion that behavior during shock, rather than uncontrollability, is the critical determinant of the observed impairment effects. There was some suggestion in both studies that shock treatments that resulted in sustained or increased intrashock activity tended to produce augmentation of fighting. Both inhibitory and facilitative effects of prior shock exposure are discussed in terms of an interacting response theory of shock treatment effects.
\end{abstract}

Recently, support has been provided for the view that levels of activity exhibited by rats during inescapable shock may be a determinant of the magnitude of subsequent impairment with escape-avoidance learning produced by such experience. Much of this evidence stems from experiments that attempted manipulations of activity levels during inescapable shock in an effort to establish the relationship between this factor and subsequent interference. One procedure involved variation in the temporal form of inescapable shock as a means of producing different levels of intrashock activity (Crowell, Lupo, Cunningham, \& Anderson, 1978; Lawry, Lupo, Overmier, Kochevar, Hollis, \& Anderson, 1978). A rapidly interrupted (i.e., pulsating) dc shock produced sustained levels of intrashock movement, whereas a noninterrupted (i.e., continuous) shock resulted in the development of relative immobility during shock. In another procedure (Anderson, Crowell, Cunningham, \& Lupo, 1971), a passive-escape paradigm was employed for one group to reduce activity levels during pulsating shock below those exhibited

\footnotetext{
Experiment 2 of this report was submitted by the third author in partial fulfillment of the requirements for the MA degree at Notre Dame. Portions of this research were presented at the meetings of the Midwestern Psychological Association in 1975 and 1978. The third author presently is at Kreuger, Green Bay, Wisconsin, and the fourth author is in the Department of Psychology, Creighton University, Omaha, Nebraska. Requests for reprints should be sent to D. Chris Anderson or Charles R. Crowell, Department of Psychology, University of Notre Dame, Notre Dame, Indiana 46556
}

by subjects in a yoked condition comparably exposed to inescapable shock. With both of these procedures, a negative relation was observed between movement during the initial shock treatment and magnitude of later interference with escape-avoidance learning. The results of these studies were interpreted by Anderson et al. (1979) as support for the view that, through learning or some related process, rats may develop immobility tendencies in the presence of shock stimuli during the initial treatment. Such reactions, if they recurred during test shock, would be expected to compete with the active responding required by the escape-avoidance task.

\section{EXPERIMENT 1}

One implication of this competitive-response interpretation is that interference should result whenever passive response tendencies develop during prior inescapable-shock exposure and active responses normally are evoked by shock during later testing. This deduction follows from the premise that active and passive response tendencies are mutually incompatible. The purpose of the present research was to evaluate this implication in the context of an active response test procedure other than escape-avoidance, the shock-elicited fighting situation, wherein impairment has been shown to follow upon prior exposure to inescapable shock (e.g., Anderson, Crowell, Lupo, \& Sarmina, 1977; Maier, Anderson, \& Liberman, 1972; Payne, Anderson, \& Murcurio, 1970; Powell, Francis, Francis, \& Schneiderman, 1972; Tondat, 1974). If 
the preceding incompatible-response conception of impairment is correct, then the procedures described above for producing variations in movement during shock would be expected to have proactive consequences for shock-elicited fighting similar to those found in the escape-avoidance learning task. In Experiment 1 , the influence of temporal form of inescapable dc shock on activity during shock and subsequent fighting was evaluated. It was expected, on the basis of previous work, that subjects exposed to an inescapable pulsating shock would exhibit relatively sustained levels of movement during shock and would show little, if any, subsequent impairment of fighting. An inescapable continuous shock, however, was expected to result in the development of immobility during inescapable shock as well as interference with later fighting.

\section{Method}

\section{Subjects}

The subjects were 30 naive male Sprague-Dawley rats obtained from Harlan Industries, Cumberland, Indiana. All were approximately 110 days old at the beginning of the study. They were individually caged, weighed daily, and maintained on a 12-h lightdark cycle. Free access to food and water was provided in the home cages throughout the experiment, and all treatment and test procedures occurred in the middle $2 \mathrm{~h}$ of the light cycle.

\footnotetext{
Apparatus

Shock treatment. A flat acrylic platform, supported on three wooden dowels over a plywood base, was used for restraining subjects (modified after Lykken \& Rose, 1959). The platform included separate relief areas for the subject's genitals, head, and limbs. One dowel was located between the forelimb holes, and one dowel was between each hindlimb hole. The platform was contained in a sound and light-resistant ventilated refrigerator shell. An Alton ultrasonic motion detector (model 5) recorded movements during each shock. The transmitting and receiving transducers of this device were mounted $9 \mathrm{~cm}$ apart upon ring stands located $17 \mathrm{~cm}$ from the subject's right side. The front (receiving) transducer was perpendicular to the head and shoulder alignment of the subject. The sensitivity of the motion detector was adjusted to a setting of 1.0 , which readily recorded gross body movements (e.g., head turning) but not slight movements (e.g., respiration). The electronic circuitry for this device was located outside the subject enclosure.

The shock source was a constant-current, tube-regulated, dc device (Campbell \& Teghtsoonian, 1958) set at $4.0 \mathrm{~mA}$, as measured with a $100-\mathrm{k} \Omega$ resistor substituted for the subject. The shock could be interrupted 10 times per second (for the "pulsating-shock" condition) with a Lehigh Valley pulse stream generator (Model 1670), which produced square-wave on-off times of $.05 \mathrm{sec}$. All treatment contingencies were controlled with solid-state equipment and a laboratory computer housed in another room.

Testing. Shock-elicited fighting tests were conducted in an acrylic chamber with inside dimensions of $15.2 \times 15.2 \times 30.4 \mathrm{~cm}$. The floor and inside front wall of this chamber were composed of $.4-\mathrm{cm}$ brass rods, spaced $1.25 \mathrm{~cm}$ apart, center to center. The wall rods were arranged vertically along the side surface of a Plexiglas sheet. The remaining three walls were lined with isolated sheets of stainless steel arranged in such a way that all grid and metal surfaces could be electrified independently. This chamber was housed in a sound-resistant, ventilated refrigerator shell with a clear plastic window that provided for observation of fighting. During testing, a 7.5-W bulb illuminated the interior of the refrigerator shell, which was located in a darkened room. The brief shocks that elicited fighting in this phase were generated by the treatment
}

source, the output of which was fed through a scrambler to the grids and walls of the test chamber. Intensity of the shock was measured at $3 \mathrm{~mA}$ with a resistor of $29 \mathrm{k} \Omega$ substituted in place of the rat. This value was selected on the basis of preliminary research indicating that it produced optimum levels of fighting.

\section{Procedure}

The study was run in five identical replications of two subjects per group.

Shock treatment. Three groups were employed. Two were restrained and exposed to either a pulsating or a nonpulsating continuous shock. In addition, a nonshock group was simply restrained for a comparable period of time. On each treatment day, subjects were exposed to light etherization (20-40 sec in a closed jar) and were restrained on the acrylic platform by a 2 -in. elastic bandage wrapped around the torso, with the front limbs taped together and the hind limbs taped separately to the adjacent dowels. Polished copper electrodes, lightly moistened with electrode paste, were attached firmly to the outstretched sole of each hindpaw with the adhesive tape. Treatment was given to each subject separately and began approximately 10 min after each etherization and $6 \mathrm{~min}$ after placement of the rat in the refrigerator shell.

Shock-treated subjects received 645 -sec shocks on each of 3 consecutive days at intervals averaging $60 \mathrm{sec}( \pm 15 \mathrm{sec})$. The nonshock control rats were treated identically to those shocked except for the exposure to shock. In the shock conditions, the number of movements was recorded during shock for each subject. Records from comparable time periods for the control subjects, however, revealed little, if any, movement.

Testing. The subjects in this study were paired within groups on a random basis, and the same pair was tested for shock-elicited fighting at approximately the same time on each of 5 consecutive days, the first beginning approximately $24 \mathrm{~h}$ following shock treatment. On each day, testing began $20-40 \mathrm{sec}$ following placement in the chamber and consisted of three consecutive test series. Each series was composed of 10 pseudoshock (i.e., no-shock) and 10 shock presentations. The purpose of the pseudoshock presentations was to determine degree of fighting to the cues accompanying presentation of shock. No fighting was observed during the pseudoshock presentations. Each shock presentation was $.5 \mathrm{sec}$ in duration, and the interval between shocks was $1.5 \mathrm{sec}$.

Scoring. Fighting was scored by a single, highly practiced experimenter who was uninformed about a subject's treatment history as well as whether a given presentation was a pseudo or shock trial. The onset of each shock (either pseudo or actual) was indicated to the scorer by an unobtrusive visual signal. At that time, a fight response was scored if one or both rats, while facing one another, attacked or made physical contact by means of either the paws or teeth. A posture response was scored if both subjects faced each other in an upright position but did not make obvious physical contact after shock onset. To evaluate the reliability of these scoring procedures, three additional persons were instructed in their use, and all independently evaluated six pairs of rats. Scoring protocols were correlated for these observers, and all interrater agreement values exceeded $90 \%$ for each of the six pairs scored.

\section{Results and Discussion}

The replications factor was not a reliable source of variance, nor did it interact with other variables. The data reported below, therefore, were collapsed across this factor.

\section{Shock Treatment}

The number of movements during shock and latency to the first movement from shock onset was averaged, for each subject, over blocks of eight pre- 


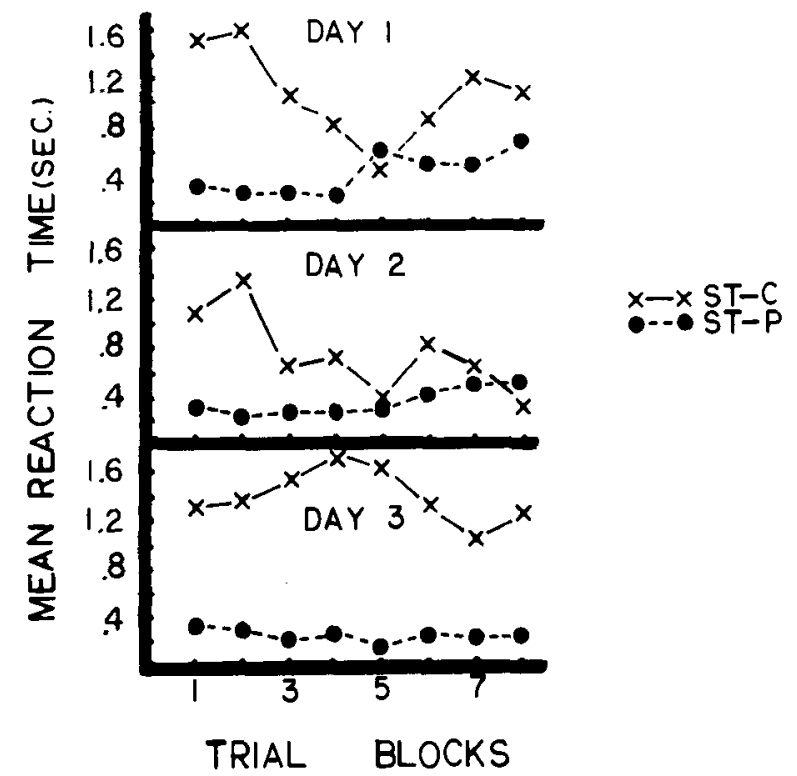

Figure 1. Mean time to the first reaction during shock for each shock-treated group in Experiment 1 as a function of blocks of eight shock presentations on each of 3 treatment days. Group STp was exposed to a pulsating shock, while Group STc received a series of continuous shocks.

sentations within a day. The mean latencies to the first movement from shock onset are presented in Figure 1 for each of the two shock-treated groups. Although on Days 1 and 2, the movement reaction times of the subjects exposed to the continuous shock treatment (STc) were consistently longer on initial trial blocks than those of pulsating shock-treated subjects (STp), the response times tended to converge over terminal blocks on these days. On Day 3, however, STc subjects exhibited consistently longer latencies than STp subjects across all trial blocks within the day.

Statistical confirmation of the pattern of effects revealed in Figure 1 was provided by separate group by block ANOVAs applied to the reaction time data for each day. Results indicated significant Groups by Blocks interaction effects on Day $1[\mathrm{~F}(7,126)=2.71$, $\mathrm{p}<.02]$ and on Day $2[\mathrm{~F}(7,126)=2.30, \mathrm{p}<.03]$, but not on Day 3. Follow-up tests for each half of each day (i.e., averaged over Blocks 1-4 or Blocks 5-8) revealed significantly greater response times for the STc than for STp subjects on the first half of each day $[F s(1,18)=5.65,9.80,8.58$, all $\mathrm{ps}<.03$, for Days 1,2 , and 3, respectively], but only for the last half of Day $3[F(1,18)=8.50, p<.01]$.

The numbers of movements during treatment shock (averaged over blocks) were subjected to analyses comparable to those applied to the response time measures. The separate group by block ANOVAs of these data revealed that STc subjects exhibited significantly less movement as a group during shock than STp rats on each treatment day [all $\mathrm{Fs}(1,18)>20.0$, $\mathrm{ps}<.01]$. No other effects were reliable in these analyses, although the Groups by Blocks interaction approached significance $(\mathrm{p}<.10)$ on Day 1 .

These findings are generally consistent with the results of previous studies manipulating the temporal form of dc shock (Crowell et al., 1978; Lawry et al., 1978). As noted, the earlier research indicated that a continuous shock promoted relatively lower levels of intrashock activity than a pulsating stimulus. In the present study, this effect was apparent both in terms of the latency to the first reaction during shock as well as the number of movements during a shock. The latency index revealed that while STc subjects exhibited relative immobility to shock onset from the start of treatment during initial daily shocks, reduced movement to shock onset during later shocks for these rats emerged only gradually over days. In contrast, STp animals exhibited consistently shorter latencies and significantly higher levels of intrashock activity than STc rats throughout treatment. These findings suggest that movement during shock, especially at its onset, for STc subjects may be influenced by different factors in the early and later portions of each treatment day. The relatively short and unchanging movement response times exhibited by these rats during initial shocks on each day may be indicative of a shock-elicited reaction, whereas the development of immobility as revealed by progressively longer latencies over days during later trials may have resulted from learning.

\section{Testing}

The percentage of fights for the 30 shock presentations of each test day was calculated for each pair of rats, and the daily median values are presented for the shock-treated and control conditions in Figure 2.

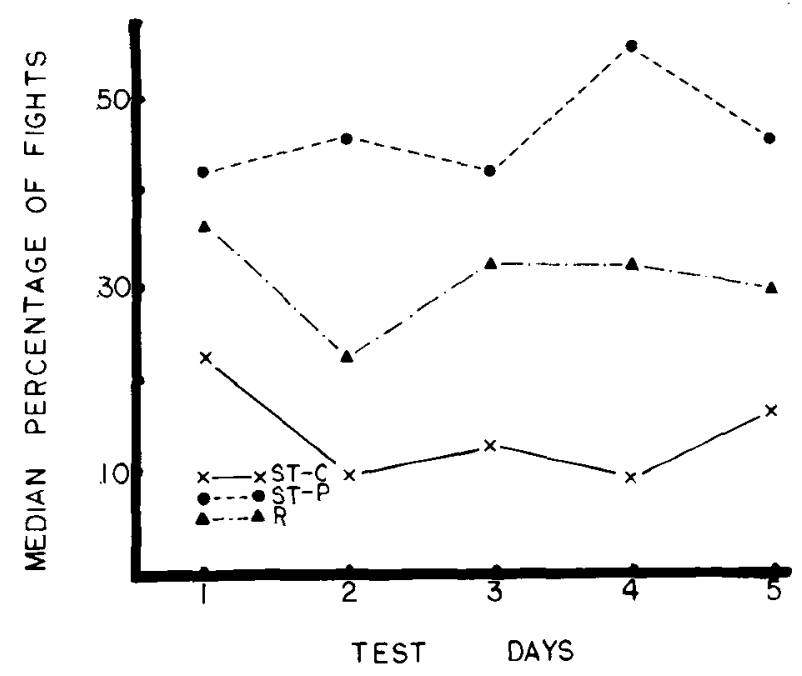

Figure 2. Daily group median percent fight responses for Experiment 1 subjects that previously were not shocked but merely restrained (R), or were exposed to pulsating (STp) or continuous (STc) shock treatments. 
It is apparent that STc subjects evinced lower levels of fighting on each day than nonshocked, restrained (R) controls. In contrast, STp rats, if anything, fought more than did controls over days.

The significance of these group differences was evaluated by means of appropriate planned pairwise nonparametric comparisons ${ }^{1}$ applied to the overall group percentage fighting levels derived from the 150 shock trials totaled across days. These comparisons revealed that the overall differences between Groups STc and STp $[U(5,5)=0, p<.01]$ and Groups STc and $R[U(5,5)=3, p<.03]$ were significant. The overall difference between Groups STp and $R$ approached significance $(p<.07)$. Thus, of the two inescapable-shock groups, only STc subjects exhibited impaired fighting behavior relative to controls during testing. Group STp displayed no indication of a debilitating effect of prior shock exposure and, in fact, showed a tendency (although not statistically significant) toward exaggerated fighting. These conclusions derived from the data of the groups over all days are fully consistent with those based upon separate planned comparisons of percentage fighting for each day, except that the difference between Groups STp and $R$ emerged significant on Days 2 and 4 (Us $\leqslant 4$, ps $<.05$ ).

The present findings are fully compatible with the general notion that the kind of behavior a subject comes to exhibit during prior shock exposure may be a critical determinant of the proactive consequences of such stimulation. In this case, as in previous studies (Crowell et al., 1978; Lawry et al., 1978), when behavior during inescapable dc shock was manipulated across groups by means of variations in the temporal form factor, differences between those groups emerged during subsequent testing that were consistently related to the disparate behaviors promoted during shock treatment. Subjects exhibiting reduced movement during the course of continuous shock exposure were subsequently impaired relative to controls when the normal behaviors observed during testing (i.e., fighting or shuttling) were incompatible with nonmovement. Alternatively, the sustained movement during treatment shock produced by the pulsating condition has been found indicative of later test performance that is similar to (or better than) that of controls. Such findings strongly suggest that the different intrashock behaviors promoted by continuous and pulsating shocks become associated with situational features of the treatment environment, such as shock stimuli, and subsequently generalize to related cues in the test situation, in which they interact with (i.e., add to or subtract from) test performance.

\section{EXPERIMENT 2}

While suggestive, the negative overall correlation between levels of activity during treatment shock and amount of subsequent shock-elicited fighting observed in Experiment 1 is not definitive evidence that the former determines the latter. Pulsating and continuous shocks differ in various physical, and possibly functional, respects, other than the kind of behavior prompted by each. Among the most notable of these is a difference in the overall amount of shock entailed by equal durations of pulsating and continuous shock. Because of its square-wave nature, a given duration of pulsating shock involves only half as much actual "shock-on" time as a comparable duration of the continuous form. Thus, a difference in total shock-on time is perfectly confounded with the disparate behaviors promoted by continuous and pulsating shock. In order to eliminate this possible confounding, as well as others (cf. Crowell et al., 1978), procedures must be employed that permit behavior to be varied across groups in the presence of otherwise physically identical treatment shocks.

One means of accomplishing this goal was employed in a study of the shuttle-interference phenomenon with rats by Anderson et al. (1979). These investigators equated two groups, by means of yoking, for exposure to a pulsating shock that normally promoted relatively high levels of intrashock activity. Movement during shock for one group was reduced, however, through the use of explicit reinforcement for immobility (i.e., passive escape), while the other yoked group received equivalent shock regardless of its movement. The results of this study showed that subjects reinforced for immobility exhibited both lower levels of intrashock movement and greater subsequent impairment of test performance than yoked counterparts, despite physically equivalent shock exposures. In the present study, similar procedures were employed to expose two groups to physically equivalent amounts of the movement-producing pulsating shock condition of Experiment 1 while encouraging nonmovement in one group through explicit reinforcement.

\section{Method}

\section{Subjects}

The rats used were naive Sprague-Dawley males obtained from the same supplier and caged and treated in the same way as the subjects of Experiment 1.

\footnotetext{
Apparatus

Shock treatment. The restraining apparatus and shock source specifications were the same as in Experiment 1. Only the pulsating shock stimulus from Experiment 1 was used. The major difference from the first study was that head panels were added to the restraint platform in order to provide subjects with more distinctive feedback for responding than that supplied by the motion detector previously employed. These panels were $7.62 \mathrm{~cm}$ long $\times 5.1 \mathrm{~cm}$ wide and were located $.65-.70 \mathrm{~cm}$ from either side of the rat's head. They formed a V-shape that conformed roughly to the curvature of the subject's head. Microswitches were activated when these panels were pressed (15 g pressure). These panels thus served in place of the motion detector as the means of
} 
recording head and upper-body movement during treatment shock.

Testing. The apparatus was the same as that used in Experiment 1 .

\section{Procedure}

This study involved three groups of 10 rats each, two shocktreated and one nonshocked control. One shocked group was exposed to a reinforcement contingency for nonmovement (i.e., passive escape), while the other received an identical pattern of inescapable shocks through a yoking procedure. The experiment was conducted in five separate replications of two subjects per group per replication. Rats were assigned to conditions in each replication using a randomized blocks procedure to equate groups for weight. All treatment and testing procedures were conducted at approximately the same time of day for each subject.

Shock treatment. The subject enclosures and methods for restraint and electrode placement were the same as those employed in Experiment 1. The procedures for this study were applied simultaneously to three subjects at a time (i.e., a triplet), one rat from each of the experimental conditions. Approximately $15 \mathrm{~min}$ after placement in the sound-attenuation chamber, identical pulsating shock treatments were administered separately to two of the three subjects in each triplet. Rats in the passive-escape condition could minimize the duration of this shock by learning not to displace the head panels for a predetermined period of time. Initially, the extent of nonresponding required to minimize shock exposure (i.e., the criterion interval) was $2 \mathrm{sec}$. If, within the maximum trial duration of $30 \mathrm{sec}$, the rat failed to respond during an interval equal to the criterion, shock was terminated immediately. However, the trial was considered "correct" only if the period of movement resulting in shock termination started with the onset of shock. If three consecutive or four out of any series of five trials were correct, the criterion interval was incremented by $1 \mathrm{sec}$. Seven consecutive incorrect trials resulted in a 1-sec decrement in the criterion interval. Shocks were delivered at intervals averaging $30 \mathrm{sec}( \pm 15)$ until each passive-escape rat achieved the maximum criterion interval of $15 \mathrm{sec}$ or until a total of 150 shocks were received.

The second shock-treated member of each triplet was exposed to the same shock delivered to the passive-escape rat but independent of movement. Collectively, these second members comprised the yoked, inescapably shocked group. The final member of each triplet served as a nonshocked control and was simply restrained during the treatment period. All subjects were administered 2 consecutive days of their respective treatments with the same criterion intervals employed each day. Shock intensity for the first day was $1.0 \mathrm{~mA}$ and for the second was $4.0 \mathrm{~mA}$. Number of panel displacements during each shock and the time to the first movement from shock onset were recorded for all shock-treated rats. The same measures for comparable nonshock periods were obtained for controls.

Testing. The general test procedure was the same as that employed in Experiment 1, except that only 2 consecutive test days, involving a total of six sets of 10 pseudoshocks and 10 shocks per day, were administered. All fighting sessions were videotaped for later scoring.

\section{Results and Discussion}

\section{Shock Treatment}

All but one subject in the passive-escape group achieved the final 15-sec criterion interval on Day 1 , the average trials to this criterion being 66.2 . On Day 2, all passive-escape rats achieved the final criterion in an average of 53.2 trials. The improvement over days for these subjects was significant $[\mathrm{t}(9)=$ $1.21, p<.05]$. In contrast, none of the yoked rats or nonshocked controls exhibited any consistent ten- dency to remain motionless during their respective treatments on either day.

Both number of movements per second of shock-on time and latency to respond from shock onset were obtained for each subject in the shock-treatment conditions at each criterion duration. Similar measures of control performance for comparable nonshock periods revealed little or no movement. Accordingly, only the treatment data of the passiveescape and yoked groups are discussed further. Furthermore, since results of the analyses of the movements per second and latency measures for the two shock-treated groups were highly similar, only the latter are presented in detail.

Mean reaction times from shock onset for each shock-treated group at each criterion interval on both treatment days are presented in Figure 3. It is apparent on both days that passive-escape subjects exhibited reaction times at many of the criterion intervals that more closely approximated theoretically perfect performance (signified by the solid diagonal line) than did yoked rats. Moreover, while passiveescape rats showed improved performance (i.e., decreased movement) on all days, especially at larger criterion intervals, yoked subjects, if anything, were more active on Day 2 than on Day 1.

An overall groups by criterion levels by days analysis indicated significant effects only for groups $[F(1,18)$ $=7.8, p<.02]$, criterion levels $[F(13,234)=109.2$, $\mathrm{p}<.01]$, and the Criterion Levels by Groups interaction $[\mathrm{F}(13,234)=3.52, \mathrm{p}<.01]$. Follow-up group tests, averaged over days, at each criterion level revealed that the interaction resulted from significant group differences on some days but not on others.

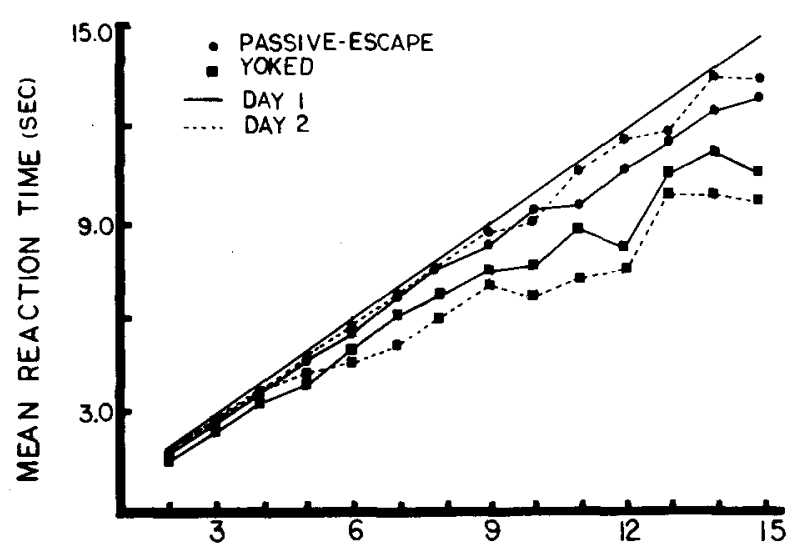

CRITERION SHOCK DURATION (SEC)

Figure 3. Mean time to the first reaction during shock on each of 2 treatment days in Experiment 2 for subjects exposed to a passive-escape contingency for nonmovement or for yoked inescapably shocked rats. The solid diagonal line represents theoretically perfect escape performance at each criterion duration. 
Specifically, passive-escape subjects exhibited significantly longer response times than yoked rats at six of the last seven criterion levels (i.e., Levels 10-15 $[\mathrm{Fs}(1,18) \geqslant 7.0, \mathrm{ps}<.01]$ but only at three of the first seven (i.e., Levels $5,7,8)[\mathrm{Fs}(1,18) \geqslant 6.0$, ps $<.03]$. Although none of the effects involving days emerged significant from the overall analysis of the response time measure, evidence indicating an improvement in performance over days was obtained with the trials-to-criterion measure described above. However, analyses of the latency measure did reveal that the performance of the passive-escape group, averaged over criterion intervals, was significantly different from theoretically perfect on Day $1[t(9)=$ $3.2, \mathrm{p}<.01]$ but not on Day 2 .

A groups by criterion level by days ANOVA was applied to the movements per second data for the passive-escape and yoked groups. Results of the main effect groups test indicated that passive-escape rats exhibited significantly lower movement rates during shock than the yoked subjects regardless of criterion level or day $[F(1,18)=15.45, p<.01]$. The only other effect to emerge significant in this analysis was the Criterion Levels by Days interaction $[\mathrm{F}(13,234)$ $=1.78, \mathrm{p}<.05]$ that resulted from a significant main effect of criterion-level effect on Day $1[F(13,117)=$ $2.34, \mathrm{p}<.01$ ] but not on Day 2 .

These findings during treatment may be taken to indicate that a passive-escape contingency is an effective procedure for increasing movement response times and decreasing overall rates of activity in the presence of a pulsating dc shock treatment. In contrast, mere exposure to the same shock without a reinforcement contingency for nonmovement did not encourage immobility and, if anything, over days tended to promote decreased latencies to movement from shock onset. This general pattern of results is comparable to that reported by Anderson et al. (1979) and Crowell and Anderson (1979) using similar procedures with rats in the context of a pulsating ac shock treatment.

\section{Testing}

The video records of shock-elicited fighting tests for each pair of subjects were independently scored on three separate occasions by practiced observers who were naive as to the group designations of each pair. The separate fighting and posturing protocols obtained were evaluated both for within-observer $(97 \%)$ and between-observers $(85 \%)$ consistency. The data reported below are based only upon those instances of common agreement between observers as to the occurrence of a posture or a fight.

The median percentages of fights in response to shock out of the 60 shock presentations for each group on each test day are shown in Figure 4. On both days, passive-escape subjects fought less and yoked rats

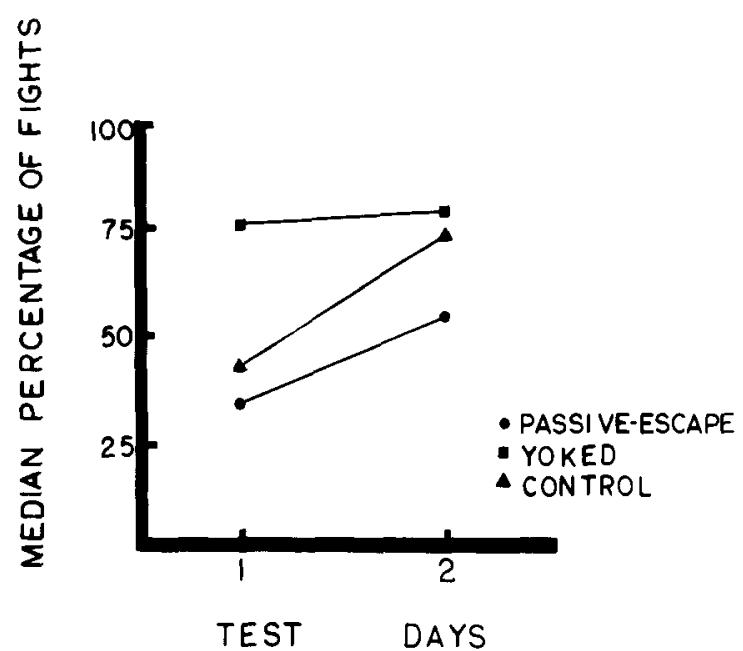

Figure 4. Daily group median percent fight responses of Experiment 2 subjects that previously received passive-escape or yoked shock treatment or that were not previously shocked (control).

fought more than nonshocked controls. An analysis of the data collapsed over both days was conducted by determining the percentage fights for each subject out of a total of 120 test shocks (i.e., Day 1 and Day 2). The median group percentages of fighting determined in this way were $42 \%, 66 \%$, and $60 \%$ for passive-escape, yoked, and controls, respectively. Planned pairwise comparisons of these percentage values indicated that the fighting of passive-escape rats was significantly impaired relative to that of controls $[\mathrm{U}(5,5)=4, \mathrm{p}<.05]$ or yoked subjects $[\mathrm{U}(5,5)=3, \mathrm{p}<.05]$. However, the comparison of yoked and controls was not reliable. While the order of group median percentages within each day was consistent with that collapsed over days, separate daily planned comparisons revealed that passiveescape rats fought significantly less than yoked subjects on each day $[U s(5,5) \leqslant 4$, ps $<.05]$ but were impaired relative to controls only on Day 2 $[\mathrm{U}(5,5)=3, \mathrm{p}<.03]$. The yoked-control comparison was reliable on neither day but approached significance on Day $1(\mathrm{p}=.10)$.

These findings may be taken as clear indication that the active, shock-elicited fighting behavior of rats can be impaired by prior exposure to a procedure designed to promote relative immobility during shock. The debilitating consequences of prior passive-escape experience might be revealed not only in reduced overall fighting, relative to previously nonshocked controls and inescapably shocked rats, but also in the pattern of any fighting that should occur. That is, not only would immobility during shock be expected to decrease the frequency of fighting in general, but it conceivably could also result in a reduced likelihood of continued fighting, given the initial occurrence of such a response. To examine this 
possibility, the video records for each subject triplet from both test days of the present study were scored to determine the number of instances that any given fight in a block of 10 shock trials was followed by consecutive "runs" of fights from 0 to 9 in length. These frequencies in each separate run-length category were expressed as a proportion of the total number of instances for a rat in all categories. The median cumulative proportions of total run lengths in each category from 0 to 9 are presented in Figure 5 for each group. In Panel A, the proportions are accumulated for run lengths less than or equal to each value from 0 to 4 , whereas Panel B presents cumulative proportions for run lengths greater than or equal to each value from 5 to 9 . In general, this figure reveals that a relatively higher proportion of the total number of consecutive fight sequences for passive-escape subjects consisted of four or less fights (i.e., short runs), and a relatively lower proportion was composed of five or more (i.e., long runs). In contrast, yoked and control rats exhibited an opposite pattern of fighting. For these subjects, a relatively lower proportion of total fight sequences were short runs and a relatively higher proportion were long runs. In fact, considering the run-length category of 9 or greater, only one pair of passive-escape rats over both days evinced a single fight sequence of this magnitude, whereas all but one of the yoked and control pairs had at least one sequence of this length.

Statistical confirmation of the pattern of effects reflected in Figure 5 was accomplished by planned pairwise comparisons at each run-length level. These tests revealed that passive-escape subjects were significantly different from yoked rats at all run-length values except 6 and 9 [Us $(5,5) \leqslant 4$, ps $<.05]$. Con-

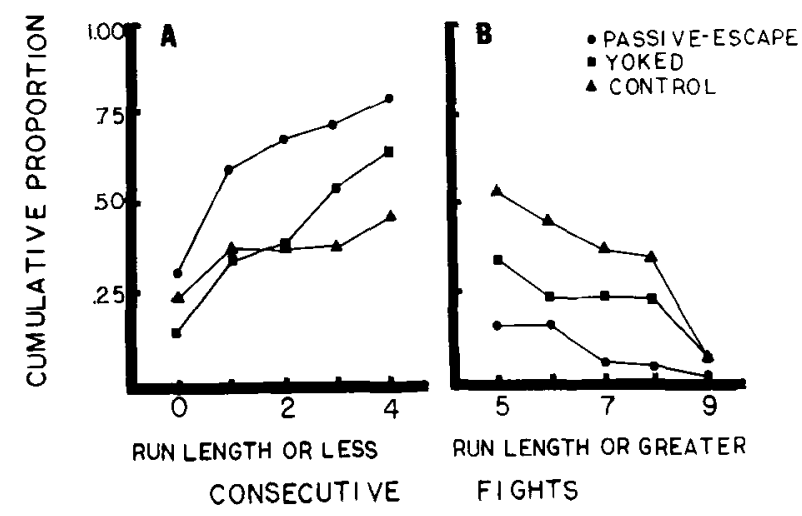

Figure 5. Group median cumulative proportion of total consecutive runs of fights as a function of run-length categories from 0 to 9 for passive-escape, yoked, and control rats in Experiment 2. Panel $A$ depicts the proportions accumulated for run lengths less than or equal to values from 0 to 4 , whereas Panel $B$ represents cumulative values for run lengths greater than or equal to values from 5 to 9. trols and passive-escape rats differed significantly at all categories except 0 and 9 [Us $(5,5) \leqslant 3$, ps $<.03$ ]. Finally, yoked and control rats differed reliably only at run-length values of 4 and $6[\mathrm{Us}(5,5)=4$, ps $<.05]$.

Taken together, these results indicate that shockelicited fighting behavior in the rat, like shuttle escape-avoidance responding (Anderson et al., 1979), is subject to impairment as a result of contingencies that promote passive-escape learning during prior shock exposure. Moreover, since shock delivered by means of a passive-escape procedure resulted in reduced intrashock activity relative to that resulting from exposure to inescapable shock, despite the physical and functional identity of the two treatments, it seems reasonable to conclude that the behaviors exhibited during prior shock are an important determinant of the subsequent impairment of fighting. Finally, the similarity of the present results to those of Experiment 1 in terms of the general relation of activity during treatment shock and fighting in response to test shock strongly suggests that similar factors were operative in both studies.

\section{GENERAL DISCUSSION}

The present findings appear to have relevance for current conceptions of proactive, shock-induced disruption of fighting behavior in the rat. One hypothesis, espoused by Maier et al. (1972), is that impairment of fighting produced by prior exposure to shock is an instance of the reportedly general phenomenon of "learned helplessness." According to the helplessness account, exposure to inescapable (i.e., uncontrollable) shock leads an organism to learn that its behavior is independent of features of the shock. Purportedly, such learning results in a cognitive state that is accompanied by associative and motivational deficits that make it difficult for an organism to respond "normally" in subsequent test situations. Fighting impairment following inescapable shock would thus be viewed as an instance of this sort of disruption.

Several aspects of the present results do not appear consistent with this interpretation of fighting interference effects. Most importantly, the impairment reported herein clearly was not dependent upon prior exposure to uncontrollable shock. In fact, it may be derived from the present studies that uncontrollability of shock is itself neither an essential nor a sufficient ingredient of shock treatment for producing subsequent fighting disruption. This conclusion follows from the observations that: (1) of the two shocktreated groups in Experiment 2, only subjects in the escapable shock (i.e., controllable-shock) condition exhibited impairment; and (2) of the two shock treatments in Experiment 1, both of which were programmed to be inescapable, only the continuous 
temporal form resulted in later interference. The only aspect of shock treatment in the present studies that appears consistently related to the occurrence of subsequent disruption is the development of some form of immobility reaction during the course of prior shock exposure.

Our findings seem most consistent with a conception recently referred to as "interacting response theory" (Anderson et al., 1979). According to this view, proactive, shock-induced interference effects are mediated by some response tendency that is acquired during prior shock exposure and subsequently generalizes to test situations in which it interacts with the behaviors normally observed there. If the interacting response tendency is incompatible with the behaviors that normally occur during testing, then interference would be expected to result. The general relation in the present data of immobility during treatment and later impairment would appear to be in accord with this implication of the interacting response account. Clearly, the development of a tendency to remain motionless during treatment shock would, if evoked by test shock, compete with fighting behavior.

Another implication of interacting response theory is that interference effects should not be necessarily dependent upon prior exposure to uncontrollable shock. Impairment would be expected to result from any shock treatment, inescapable or otherwise, that was accompanied by the development of response tendencies incompatible with subsequent test performance. As noted above, the present results are in accord with this expectation. Taken together, the results of both studies are that the reaction a subject comes to exhibit during treatment is a better indicator of whether or not fighting impairment will occur than are features of the prior shock stimulus such as its uncontrollability.

Also, it must be noted that proactive effects of shock treatment other than interference logically are possible within the framework of an interacting response account. Not all response tendencies that may develop during shock treatment would be expected to impair subsequent performance, given that they recur during testing. In support of such a possibility, Glazer and Weiss (1976) have shown that some previously developed responses to shock actually facilitate rather than retard test performance. Certain aspects of the present data appear to represent a similar augmentation effect through prior exposure to shock. In Experiment 1, rats exposed to an inescapable pulsating shock exhibited consistently high levels of intrashock movement throughout treatment and subsequently fought significantly more than controls on some test days. Similarly, yoked subjects in Experiment 2 evinced increased movement during shock over treatment days and also fought more (although not quite significantly so) than controls.
Presumably, in these instances, the consistently high or increased activity exhibited during treatment shock may be viewed as a response tendency that, if it occurred during testing, would interact with fighting in a facilitative manner.

Finally, it must be acknowledged that the present results, particularly those of Experiment 2, conflict with earlier findings in support of the helplessness account reported by Maier et al. (1972). In their study, a yoking procedure was used to equate two groups for exposure to a continuous ac shock stimulus while allowing one to escape from shock by means of an active, wheel-turn response. Subsequently, both groups and a nonshocked control were given shock-elicited fighting tests. Thus, like Experiment 2 above, this study involved a comparison of the proactive consequences for fighting of exposure to controllable and uncontrollable shock. Unlike the present findings, however, impairment of fighting, relative to controls, was observed only for the rats in the yoked condition. One possible reason for this disparity may be the continuous temporal form of the ac shock treatment employed by Maier et al. (1972). When programmed to be inescapable, this stimulus has been shown by Lawry et al. (1978) to be accompanied by a marked reduction in intrashock activity, much like the continuous shock and the passive-escape condition of the present report. Accordingly, the subjects in the yoked condition of the Maier et al. study may have acquired an immobility response tendency during treatment that proved to be incompatible with later fighting. On the other hand, the wheel-turn escape contingency for the controllably shocked rats may have offset the normal development of immobility through the reinforcement of active responding. While speculative, the present argument suggests that the outcome of the Maier et al. (1972) study may have been consistent with the different intrashock movement levels generated by their respective treatment conditions. In this context, it must be emphasized that the present research, as well as previous studies (Anderson et al., 1979; Crowell et al., 1978; Lawry et al., 1978), strongly suggests that the choice of shock parameters, such as temporal form, may be critical in determining the proactive consequences of a shock treatment largely because of the behavior promoted during shock.

\section{REFERENCES}

Anderson, D. C., Crowell, C. R., Cunningham, C. L., \& LuPo, J. V. Behavior during shock as a determinant of the magnitude of interference with escape-avoidance learning in the rat. Journal of Experimental Psychology: Animal Behavior Processes, 1979, 5, 243-257.

Anderson, D. C., Crowell, C. R., Lupo, J. V., \& Sarmina, T. The effects of sex and exposure to inescapable shock on shockelicited fighting in albino rats. Physiological Psychology, 1977, $5,445-448$. 
Campbell, B, A., \& Teghtsoonian, R. Electrical and behavioral effects of different types of shock stimuli on the rat. Journal of Comparative and Physiological Psychology, 1958, 51, 185-192.

Crowfll, C. R., \& Anderson, D. C. Shuttle interference effects in the rat depend upon activity during prior shock: A replication. Bulletin of the Psychonomic Society, 1979, 14, 413-416.

Crowell, C. R., Lupo, J. V., Cunningham, C. L., \& Anderson, D. C. Temporal form of shock is a determinant of magnitude of interference with escape-avoidance learning produced by prior exposure to inescapable shock. Bulletin of the Psychonomic Society, 1978, 12, 407-410.

Glazer, H. I., \& Weiss, J. M. Long-term interference effect: An alternative to "learned helplessness." Journal of Experimental Psychology: Animal Behavior Processes, 1976, 2, 202213.

Lawry, J. A., Lupo, V., Overmier, J. J., Kochevar, J., Hollis, K. L., \& ANDERSON, D. C. Interference with avoidance behavior as a function of qualitative properties of inescapable shocks. Animal Learning \& Behavior, 1978, 6, 147-154.

LyKKFN, D. T., \& Rose, R. A rat-holder with electrodes for GSR measurement. American Journal of Psychology, 1959, 72, 621-622.

Maier, S. F., Anderson, C., \& Lieberman, D. A. Influence of control of shock on subsequent shock-elicited aggression.
Journal of Comparative and Physiological Psychology, 1972, 81, 94-100.

Payne, R., Anderson, D. C., \& Murcurio, J. Preshock-produced alterations in pain-elicited aggression. Journal of Comparative and Physiological Psychology, 1970, 71, 258-266.

Powell, D. A., Francis, M. J., Francis, J., \& Schneiderman, $\mathrm{N}$. Shock-induced aggression as a function of prior experience with avoidance, fighting, or unavoidable shock. Journal of the Experimental Analysis of Behavior, 1972, 18, 323-332.

Tondat, L. M. Is the effect of preshock treatment on shockelicited aggression independent of situational stimuli? Psychological Record, 1974, 24, 409-417.

\section{NOTE}

1. The method of planned comparisons was used here and in the subsequent study because specific a priori predictions, based on previous research, were made concerning the direction of the proactive effects of the passive-escape and yoked shock treatments. Accordingly, for each such comparison, an $\alpha=.05$ was employed in the interest of minimizing Type II errors.

(Received for publication June 25, 1979; revision accepted June 13,1980 .) 